\title{
Mammography Classification Based on Convolutional Neural Network
}

\author{
Changjiang Zhang and Huanhuan Nie* \\ ZheJiang Normal University, ZheJiang Province, CHINA \\ ${ }^{*}$ Corresponding author
}

\begin{abstract}
Limited by various conditions, the features of mammography images are difficult to extract, so it is hard to classify them. The paper proposed a method based on deep learning method to classify benign and malignant mammography images. The convolutional neural network concludes four convolution layers, four pool layers, and two full-connection layers, and a Softmax layer. The paper designed a new network architecture to improve the traditional one. As a result, we have done an experiment on the DDSM database. Compared with other classification methods, it shows that the method proposed in this paper is more effective than other methods.
\end{abstract}

Keywords-mammography; classification; convolutional neural network; DDSM

\section{INTRODUCTION}

January 25, 2016, the highest global impact factor Journal (CA:A Cancer Journal for Clinjicians) ( $\mathrm{IF}=144.8)$ published online 2015 article of China cancer statistics(1). The paper points out the cancer has become the first cause of disease in China, and the Morbidity and mortality are still rising, and cancer has become a very important public health problem. Among these, Breast cancer has become the most common tumor in Chinese women, which takes up the $15 \%$ proportion of all women's cancer. According to statistics, the early cure rate of breast cancer is $97 \%$, and the cure rate is only $40 \%$ after progress. Therefore, early detection, early diagnosis and early treatment are the key to improve the curative effect and reduce the mortality of breast cancer(2).

There are many methods to classify breast images. Choi et al. used LBP feature extraction method, then used SVM as classifier to classify breast images into benign and malignant ones(3). gargouri et al. combined GLLD with ANN to classify breast ROI into benign and malignant ones4. Han Zheng zhong et al. extracted morphological features of breast ROI images, combined with non-sampling wavelet transform and Gray level co-occurrence matrix. SVM is then used to distinguish between geometric and texture features Benign and malignant masses. M Hussain et al. $(5,6)$ combines Gabor features and then uses SVM to classify.

The depth learning of convolution neural network has become one of the most powerful machine learning tools Image classification exceeds almost all other classification accuracy. Traditional classification methods even human abilities $(7,8)$. The convolution process can simplify the inclusion Millions of pixels to a set of small feature maps, thus reducing Enter the dimensions of the data while preserving the most important.
Application of neural network in classification Mammography is not entirely new. However, most of the work Focus on the classification of small patches, known as regions Interest (return on investment) (9). An ROI is the region that is likely to contain a tumor. This is typically carved out of the whole images based on either clinical information or automatic segmentation. Daniel Lévy et al. used deep CNNs on small patches of mammograms, achieving a maximum accuracy of 93\%(10). Neeraj Dhungel et al. built a deep learning based method that automatically segments the area of lesions and then classifies the mammogram. The best results are total image 0.74 and total image 0.8 add the automatically detected Lessing to the whole image patches, and 0.91 for whole image plus manually segmented small patches in terms of auROC(11). Generally speaking, the classification of mammograms using small abnormality patches affords reasonable performance but requires very extensive pre-processing work.

Most of the proposed methods involved CNNs but in a traditional way, where they use only the extracted CNN features or combine them with some other hand-crafted descriptors to carry out the classification task $(12,13)$. However, the most interesting aspect of using CNNs is the end-to-end supervised learning process which does not rely on complex engineered descriptors and instead uses the whole raw image(14).

In this paper, we propose a new method classification of benign and malignant mammography images based on convolution neural network model. This paper introduces the adopted model and its parameters in detail, and makes experiments on DDSM database, and then makes the analysis of results.

\section{The Convolution Neural Network Designed IN THIS PAPER}

\section{A. Structure Diagram of Network}

The classical convolution neural network is alternately connected by winding layer and pool layer. Because of its strong robustness to translation, scaling and other deformation forms, it has been widely used in the field of image recognition. The basic structure of the convolution neural network for mammography recognition designed in this paper is shown in figure I. 


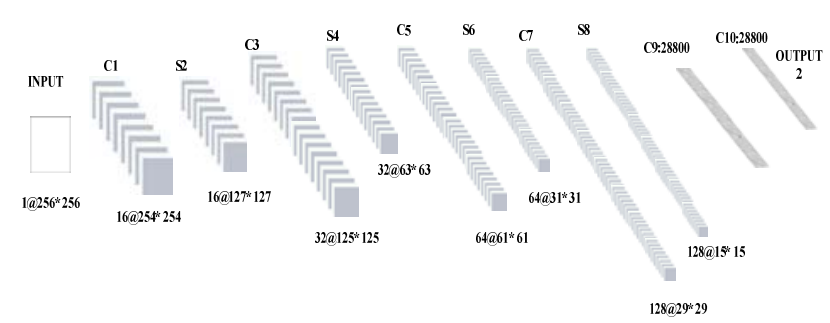

FIGURE I. CONVOLUTION NEURAL NETWORK STRUCTURE DIAGRAM.

It is mainly composed of 4 volumes, 4 pools, 2 fully connected layers and a Softmax regression layer. Among them, the first 10 layers of the network are used for feature extraction, and the last layer is used for classification.

\section{B. Detailed Configuration of Network}

To prevent overfitting, we add the Dropout layer, the detailed configuration and training parameters of the network are shown in Table I.

TABLE I. DETAILED CONFIGURATION AND NUMBER OF PARAMETERS OF CNN NETWORK

\begin{tabular}{|c|c|c|c|}
\hline $\begin{array}{c}\text { serial } \\
\text { number }\end{array}$ & Layer type & Output size & processing method \\
\hline 1 & Input layer & $256 \times 256 \times 1$ & \\
\hline 2 & $\begin{array}{l}\text { Convolution } \\
\text { layer } 1\end{array}$ & $254 \times 254 \times 16$ & $3 \times 3$ convolution \\
\hline 3 & $\begin{array}{c}\text { Activate function } \\
\text { layer 1(ReLU) }\end{array}$ & $254 \times 254 \times 16$ & \\
\hline 4 & Pool layer1(max) & $127 \times 127 \times 16$ & $2 \times 2$ maximum sampling \\
\hline 5 & $\begin{array}{l}\text { Convolution } \\
\text { layer } 2\end{array}$ & $125 \times 125 \times 32$ & $3 \times 3$ convolution \\
\hline 6 & $\begin{array}{c}\text { Activate function } \\
\text { layer 2(ReLU) }\end{array}$ & $125 \times 125 \times 32$ & \\
\hline 7 & Pool layer2(max) & $63 \times 63 \times 32$ & $2 \times 2$ maximum sampling \\
\hline 8 & $\begin{array}{l}\text { Convolution } \\
\text { layer } 3\end{array}$ & $61 \times 61 \times 64$ & $3 \times 3$ convolution \\
\hline 9 & $\begin{array}{c}\text { Activate function } \\
\text { layer 3(ReLU) }\end{array}$ & $61 \times 61 \times 64$ & \\
\hline 10 & Pool layer3(max) & $31 \times 31 \times 64$ & $2 \times 2$ maximum sampling \\
\hline 11 & $\begin{array}{c}\text { Convolution } \\
\text { layer } 4\end{array}$ & $29 \times 29 \times 128$ & $3 \times 3$ convolution \\
\hline 12 & $\begin{array}{c}\text { Activate function } \\
\text { layer 4(ReLU) }\end{array}$ & $29 \times 29 \times 128$ & \\
\hline 13 & Pool layer4(max) & $15 \times 15 \times 128$ & $2 \times 2$ maximum sampling \\
\hline 14 & Flatten layer & $1 \times 28800$ & \\
\hline 15 & $\begin{array}{c}\text { Full connection } \\
\text { layer } 1\end{array}$ & $1 \times 28800$ & \\
\hline 16 & $\begin{array}{c}\text { Activate function } \\
\text { layer } 5(\mathrm{ReLU})\end{array}$ & $1 \times 28800$ & \\
\hline 17 & $\begin{array}{c}\text { Dropout } \\
\text { layer1 }(0.5)\end{array}$ & $1 \times 28800$ & \\
\hline 18 & $\begin{array}{c}\text { Full connection } \\
\text { layer } 2\end{array}$ & $1 \times 28800$ & \\
\hline
\end{tabular}

TABLE I, cont.

\begin{tabular}{|c|c|c|c|}
\hline 19 & $\begin{array}{c}\text { Activate function } \\
\text { layer 6(ReLU) }\end{array}$ & $1 \times 28800$ & \\
\hline 20 & $\begin{array}{c}\text { Dropout } \\
\text { layer2(0.5) }\end{array}$ & $1 \times 28800$ & Classifier \\
\hline 21 & Softmax & $1 \times 28800$ & \\
\hline
\end{tabular}

The activation function used in the paper is the modified linear unit $(\operatorname{ReLU}) \mathrm{f}(\mathrm{x})=\max (0, \mathrm{x})$; the output uses a Softmax regression as a classifier with a strong nonlinear classification ability and a fast classification speed, and uses the cross entropy to banlance the loss of training. For classes of m, each class of data sets with $\mathrm{n}$ samples is in the form of.

$$
Q(\alpha)=-\frac{1}{n}\left[\sum_{i=1}^{n} \sum_{j=1}^{m} 1\left\{y^{(i)}=j\right\} \ln \frac{e^{\alpha_{j}^{\mathrm{T} x(i)}}}{\sum_{j=1}^{\mathrm{m}} \mathrm{e}^{\alpha_{\mathrm{j}}^{\mathrm{T}} \mathrm{x}(\mathrm{i})}}\right]
$$

In the formula above, the ${ }^{\alpha}$ represents the model parameter, the $1\{$.$\} is the loss function, and use the Back Propagation(BP)$ algorithm to tune-up the weights in the network. This paper reshape the original images to the images of size of $256 \times 256$, and put the images to the CNN network we proposed, through the computation of four convolutional layers and four maxpooling layers, we get 128 feature maps of size of $15 \times 15$, and then we reshape the feature maps to a vector and then connect a full-connection layer.

From the point of view of feature extraction, the output of the full connection layer is the high-level feature representation of the input image, and then output the result to the Softmax regression layer as the input layer. Through several comparative experiments, the number of neurons in the final connection layer is 28800 .

\section{EXPERIMENT}

\section{A. Experiment Data}

The experiment data of this paper comes from the DDSM database. The DDSM database is a high-resolution standard database provided by the southern University of Florida and built in 1999 to study mammographic mammography. In the database, the focus areas have been marked by specialized medical experts, and the database also includes diagnostic conclusions, which includes The conclusion of benign and malignant diagnosis and the type of the lesion (such as the lump and calcification point). There are 2620 mammography images in this database.

It consists of 695 normal images (no lesions), 914 malignant tumor images, and 1011 benign tumor images. Each case in the database includes four images, which are two breast images taken from different angles, the two directions are CC View and MLO View respectively.

In this paper, we used the ROI(Region of interest) area of the original image marked by experts as the experimental image, the number of benign and malignant images are respectively 600 , and then expanded the datasets to a certain 
number. The original image is processed with the horizontal mirror direction, the up and down mirror directions, the direction of rotation 90 degrees, the rotation 180 degrees and the rotation 270 degrees methods. The data sets are divided into training sets and test sets respectively, and the number ratio of the training set and the test set is 4 to 1 , that is, the training set are 5760 of malignant and benign we get at last, the test set is a total of 1440 images, and the numbers of malignant and the benign are the same. The experimental images are shown in the Figure II.
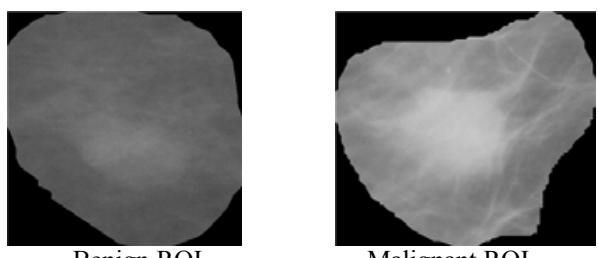

Benign ROI

Malignant ROI

FIGURE II. BENIGN AND MALIGNANT IMAGE IN DATA SET.

\section{B. Experimental Platform}

As the datasets used in this experiment is large, and it needs to obtain many parameters, we take the GPU technique to accelerate the speed. The experiment platform is a deep learning framework Tensorflow as the experiment framenwork, which bases on the Python language. The hardware the paper used is Intel i5-2400, NVIDIA GTX1050Ti, the operation system is Linux, the software we have installed are the CUDA8.0, CUDNN6.0, python of version3.6 and the Tensorflow version is 1.3.0.

\section{Experimental Flow}

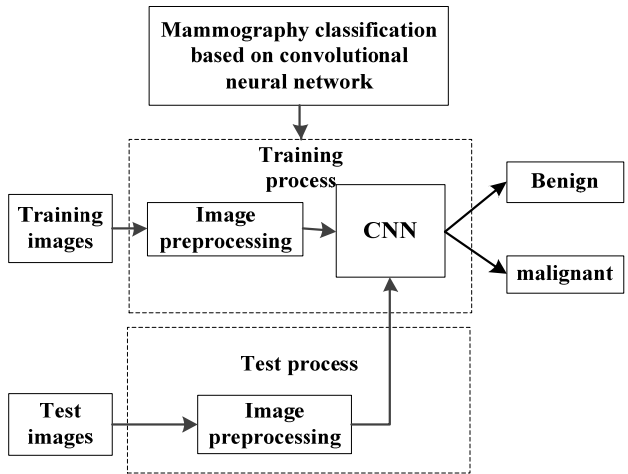

FIGURE III. MAMMOGRAPHY CLASSIFICATION FLOW CHART BASED ON CNN

As we can see in figure III, firstly, we cut the training set into the size of 256x256 in the preprocessing, and then we train a classification model through the network designed. The test set will be processed same as training set, and then test the test set by model we have got.

\section{EXPERIMENTAL RESULTS AND ANALYSIS}

The experimental results and analysis will be interpreted as follows:

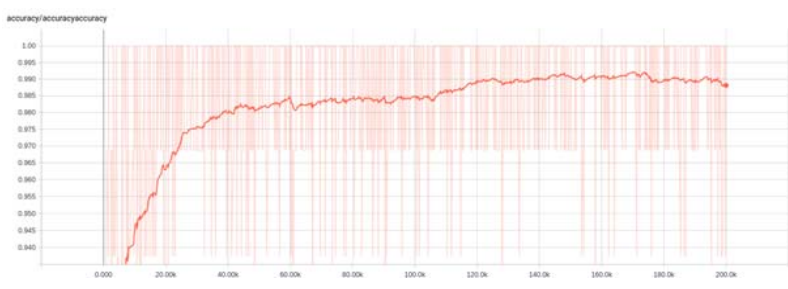

FIGURE IV. THE CHANGE CURVE OF THE RECOGNITION RATE.

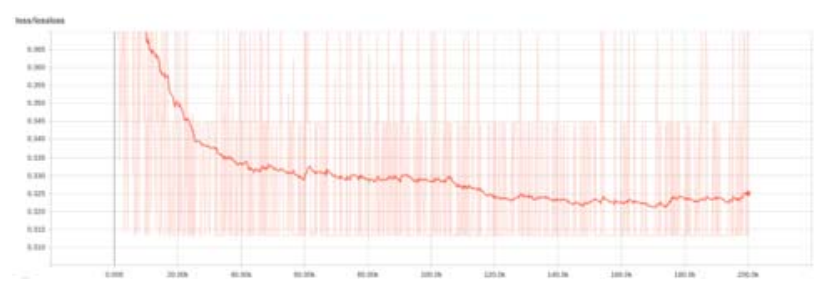

FIGURE V. THE CHANGE CURVE OF LOSS FUNCTION

The test of this experiment is based on the experimental image above. The training iterations are set to 200000 times. The curve eventually tends to converge, and tests are performed on the test set. The accuracy rate is $95.25 \%$.

TABLE II. EVALUATION PARAMETERS

\begin{tabular}{|c|c|c|}
\hline Number & Positive & Negative \\
\hline 0 & TP & FN \\
\hline 1 & FP & TN \\
\hline
\end{tabular}

$$
\mathrm{TPR}=\mathrm{TP} /(\mathrm{TP}+\mathrm{FN})
$$

$$
\mathrm{FPR}=\mathrm{FP} /(\mathrm{FP}+\mathrm{TN})
$$

Using FPR as the abscissa, TPR as the ordinate to obtain the ROC curve, AUC refers to the area under the ROC curve, so $0.5 \leq \mathrm{AUC} \leq 1$, the larger the AUC area is, the better the classification effect will be. The ROC curve obtained from the experiment is as follow.

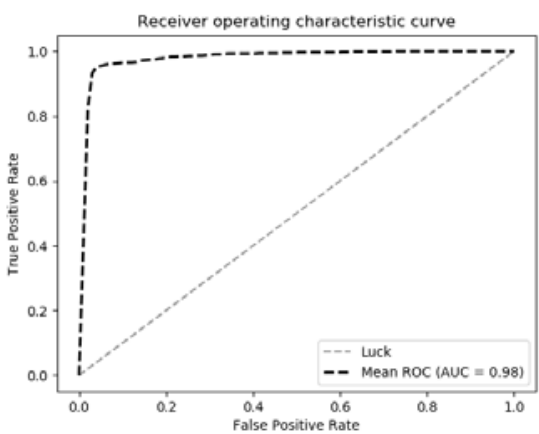

FIGURE VI. ROC CURVE

From figure VI, it can be seen that the AUC value of the experiment reached 0.98, which indicates that the classifier of 
the model training of this experiment works well. To further illustrate the effectiveness of this method, it is compared with the experimental methods of other similar images. We compare ours with other classification methods of the same kind of image, the results is as Table II.

TABLE III. COMPARISON OF DIFFERENT METHODS OF RECOGNITION RATE.

\begin{tabular}{|c|c|c|c|}
\hline Works & Database & Technique & $\begin{array}{c}\text { Accuracy of } \\
\text { classification }\end{array}$ \\
\hline Choi et al. [3] & DDSM & LBP+SVM & $82.25 \%$ \\
\hline Gargouri et al. [4] & DDSM & GLLD +ANN & $90.44 \%$ \\
\hline Han et al. [5] & DDSM & UWT+GLCM+SVM & $88.90 \%$ \\
\hline Hussain et al. [6] & DDSM & Gabor+ SVM & $94.92 \%$ \\
\hline $\begin{array}{c}\text { M. G. Ertosun et } \\
\text { al.[15] }\end{array}$ & DDSM & CNN & $85 \%$ \\
\hline Proposed & DDSM & Proposed & $95.25 \%$ \\
\hline
\end{tabular}

The data sets used in the experiment comparison are ROI images labeled by doctors in the DDSM database. The first four comparison methods firstly extract the image features, and then input the features into the classifier for classification, which requires complicated feature extraction and dimension reduction links. In the process of retraining and recognizing, there is no need for manual intervention. The original ROI image is input into the network designed in this paper. The network carries out autonomous learning features and is an end-to-end method. As can be seen from the parameters in Table 3, the AUC value of the proposed method is relatively high, and the accuracy rate can reach $95.25 \%$. Overall, this method has certain advantages in the benign and malignant classification of mammography images.

\section{CONCLUSION}

In this paper, we propose a classification of benign and malignant mammography images based on convolutional neural networks. This convolutional neural network consists of four convolutional layers, four pooled layers, two fully connected layers, and one Softmax regression layer composition. In the training of the model, in order to avoid overfitting, data amplification was used and the Dropout layer was added after the fully connected layer. Experiments were done on the DDSM database, and the method was compared with some similar image classification methods.

It shows that this method has certain advantages. However, because deep learning has certain requirements for experimental equipment, and experiments need to build and configure the network, it takes a certain amount of energy. At the same time, there will be some differences in the length of time used for the experiment. Future work attempts to use other deep learning network methods, and even different networks can be cross-connected and integrated to further improve the model's operational efficiency and recognition accuracy.

\section{REFERENCES}

[1] CHEN W,ZHENG 1L BAADE $P$ D,et al.Cancer statistics in China,2015[J].CA:Acancer Journal For Clinicians,2016, 66(2):115-32.
[2] SIEGEL R L,MILLER K D,JEMAL A.Cancer statistics,2016[J].CA:A Callcer Journal For Clinicians,2016,66(1):7-30.

[3] J. Choi, Y. M. Ro, Multiresolution local binary pattern texture analysis combined with variable selection for application to false-positive reduction in computer-aided detection of breast masses on mammograms,Phys. Med. Biol. 57 (2012) 7029-7052.

[4] N. Gargouri, A. D. Masmoudi, D. S. M. R. Abid, A New GLLD Operator for Mass Detection in Digital Mammograms, International Journal of Biomedical Imaging (2012) 1-13.

[5] Han Zhen-zhong, Liu Pei-guo, Mao Jian, A Novel Method of Extracting and Classifying the Features of Masses in Mammograms, 2017 12th International Conference on Computer Science and Education (ICCSE).

[6] M. Hussain, S. Khan, G. Muhammad, et al., Effective Extraction of Gabor Features for False Positive Reduction and Mass Classification in Mammography, Applied Mathematics and Information Sciences 8 (1L)(2014) 397-412

[7] Krizhevsky, A., I. Sutskever, and G.E. Hinton. Imagenet classification with deep convolutional neural networks. in Advances In Neural Information Processing Systems. 2012.

[8] Zhang, Y., et al. DeepSplice: Deep classification of novel splice junctions revealed by RNA-seq. in Bioinformatics and Biomedicine (BIBM), 2016 IEEE International Conference on. 2016. IEEE.

[9] Hua, K.-L., et al., Computer-aided classification of lung nodules on computed tomography images via deep learning technique. OncoTargets and therapy, 2015. 8 .

[10] Lévy, D. and A. Jain, Breast mass classification from mammograms using deep convolutional neural networks. arXiv preprint arXiv: $1612.00542,2016$.

[11] Dhungel, N., G. Carneiro, and A.P. Bradley. Fully automated classification of mammograms using deep residual neural networks. in Biomedical Imaging (ISBI 2017), 2017 IEEE 14th International Symposium on. 2017.IEEE.

[12] J. Arevalo, F.A. González, R. Ramos-Pollán, J.L. Oliveira, M.A. Guevara Lopez,Representation learning for mammography mass lesion classification with con-volutional neural networks, Comput. Methods $\begin{array}{llll}\text { Programs } & \text { Biomed. } & 127 & \text { (2016)248-257, doi: }\end{array}$ 10.1016/j.cmpb.2015.12.014

[13] Z. Jiao, X. Gao, Y. Wang, J. Li, A deep feature based framework for breast masses classification, Neurocomputing 197 (2016) 221-231, doi: 10.1016/j.neucom.2016.02.060 .

[14] Y. LeCun, K. Kavukcuoglu, C. Farabet, Convolutional networks and applications invision,

Proc.2010IEEEInt.Symp.CircuitsSyst.,2010,pp.253256,doi:10.1109/ISCAS.2010.5537907 .

[15] M. G. Ertosun, D. L. Rubin, "Probabilistic visual search for masses within mammography images using deep learning", Bioinformatics and Biomedicine (BIBM) 2015, pp.1310-1315, November 2015. 